\title{
Review
}

\section{Multidisciplinary of anti-COVID-19 battle: from immunological weapons to ecological interventions}

\author{
Federico Boem ${ }^{1}$, Giulia Nannini ${ }^{2}$, Amedeo Amedei $^{2,3, *}$ \\ ${ }^{1}$ Department of Literature and Philosophy (DILEF), University of Florence, 50121 Florence, Italy, ${ }^{2}$ Department of \\ Experimental and Clinical Medicine, University of Florence, 50134 Florence, Italy, ${ }^{3}$ SOD of Interdisciplinary Internal \\ Medicine, Azienda Ospedaliera Universitaria Careggi (AOUC), 50134 Florence, Italy
}

\section{TABLE OF CONTENTS}

\author{
1. Abstract \\ 2. Introduction \\ 3. A wider, different look \\ 4. SARS-CoV-2 and the other causes of COVID-19 pandemic \\ 5. Viruses and pandemics from an ecological standpoint \\ 6. Infections and microbiota \\ 7. COVID-19 and microbiota \\ 8. Ecological perspective in medicine and future directions \\ 9. Conclusions \\ 10. Author contributions \\ 11. Ethics approval and consent to participate \\ 12. Acknowledgment \\ 13. Funding \\ 14. Conflict of interest \\ 15. References
}

\section{Abstract}

The COVID-19 pandemic is not just a medical and epidemiological problem. In fact, its impact concerns numerous aspects of human life (such as social and the political-economic dimension). This review aims at highlighting some crucial and neglected aspects of the pandemic in order to include them into a more general framework for the understanding of the phenomenon. Accordingly, it is structured as follows. First, after e brief recap of COVID-19 onset, it is argued the so-called proximate causes of the pandemic, i.e., the mechanisms by which viruses infect their hosts and the patterns of spread of the resulting pathologies, are not enough for a more adequate understanding of it. Second, it is shown how possible solutions to the risk of an upcoming pandemic involve studying the ultimate causes of this phenomenon. This means understanding not only how COVID-19 has become a global issue but also why it was possible for this to happen. Next, it is argued that is urgent to go to the root of the possible conditions: thus looking at the ecological dimension of diseases, the role of microorganisms in evolution, up to rethinking the organiza- tion of health systems. Third, to keep these very different perspectives together entails the study of COVID-19 from the point of view of the relationships between biological entities in a purely systemic dimension. Fourth, special attention is given to the symbiotic perspective offered by the study of the microbiota. It is argued how this perspective on microbiota provides an innovative interpretative lens with which to analyze various aspects (from the immunological to the ecosystemic one) of the pandemic. In conclusion, it is claimed that this field of study could perhaps offer not only elements that will be useful to make the treatment and containment strategies of the pandemic effective in its mechanisms, but also may suggest innovative elements for the solutions about the deep reasons that have made COVID19 a global issue.

\section{Introduction}

The pandemic known as COVID-19 is certainly and primarily a global public health problem. However, its impact and consequences (from ecological factors to economic and sociopolitical aspects) go beyond that. Accord- 
ingly, understanding the pandemic would require different approaches, also from different disciplines other than clinical sciences. Indeed, by considering the numerous aspects of the phenomenon, it should not surprise how it could be differently addressed from the perspective of diverse (yet close) disciplines. As a matter of fact, epidemiology, virology and microbiology are certainly central. Therefore, the initial response to the pandemics (in the absence of tailored therapies) has been focused on the use of treatments developed for other conditions but which could still generate an immune response (the first attempts at plasmapheresis are also associated with this idea). For understandable reasons, the next effort, in constructing an effective response to the pandemic problem, focused on vaccine development [1].

Vaccines, in dealing with this issue, constitute an engaging case for, at least, two reasons. On the one hand, they have been (and still are) the subject of a fierce debate, involving the public, causing an ethical-political clash often resulting in a phenomenon known as "vaccine hesitancy" $[2,3]$. On the other hand, the urgency to develop a vaccine for SARS-CoV-2 has also changed the nature of doing research (the often-called problem of "fast-science") in ways that involved field experts on issues of a purely methodological nature [4].

However, by borrowing a famous distinction elaborated by Ernst Mayr, if vaccines are definitely a response to the proximate causes of the ongoing pandemic, they do not address its ultimate causes [5].

By proximate cause, we mean both the understanding of the development of the pandemic (through epidemiological models) and the biological mechanism that makes coronavirus infection possible (through molecular research). Unravelling these aspects remains crucial and necessary since it answers a "how" question. This means understanding how a certain phenomenon is developed (in this case the epidemic/pandemic).

On the other hand, these types of answers say nothing about the "why" question. In detail, the comprehension of the dynamics of the phenomenon leaves a question open, concerning why such a phenomenon (the epidemic/pandemic) actually takes place (implying its evolutionary and ecological roots). Investigating the reason for the pandemic, therefore, means understanding its ultimate causes.

According to this, ecology also entered with preponderance among the scientific sources to solve the problem. It is no mystery that the violence and the novelty of new pathogens are also the results of neglect and ignorance of the delicate relationships between living beings and the environment [6-10]. In other words, the ecological perspective offers a plausible explanation concerning why COVID-19 pandemics actually took place.

A link between these apparently distant areas of scientific investigation could be the microbiota. In fact, human-associated microbiota, especially gut microbiota, is now recognized as a key component of immunity (in terms of maturation, training, and functioning of the immune system) [11-13]. In addition to that, microbiota's activities depend also on its composition and ecological interaction with the host, thus suggesting that ecological investigations on micro-organismic functioning will soon be a central issue in immunology itself [7, 14-16]. Moreover, microbiota activities and biodiversity also depend on external factors, such as diet and lifestyle, which inevitably link it to environmental and socio-economic factors [17]. Because of that, the COVID-19 pandemic is not just a medical or epidemiological problem, but also a multi-level issue that requires a multidisciplinary approach in order to be solved [17].

If these multidisciplinary measures, born out of the emergency, will be successful, it is not too daring to think that they can contribute to redefining key concepts such as those of health, disease, and lastly immune response.

\section{A wider, different look}

To fully understand the ecological dimension of the pandemic, it is essential to remember that SARS-CoV-2 is a zoonosis or any infection that can be transmitted from other metazoans to humans. This transmission can occur directly (meaning that the infected species can openly pass the pathogen to humans) or indirectly (through other vector organisms). The phenomenon known as spillover occurs when a population of a given species, which presents specific pathogens, encounters a new host population of a different species, and part of this potential pathogen adapts to the new species causing a new disease. Spillover is a common occurrence; in fact, over two-thirds of human viruses display this kind of origin $[6,7,9,18,19]$.

For example, known diseases that have marked the history and development of the human species (such as smallpox, bubonic plague, and cholera) are of animal origin Fig. 1. Even the HIV virus, responsible for AIDS, is actually of zoonotic origin as it derives from non-human primates. During the spillover, the original virus can undergo modifications due to various evolutionary factors (such as different selective pressure) and the new context. This often causes the new virus to differ from the original one, generating a new disease that becomes proper to the new organism $[6,7,9,18,19]$.

This phenomenon should not surprise us and indeed reminds us not only that all living forms are connected to each other as descendants from a single common ancestor.

Furthermore, according to a line of studies that dates back to Lynn Margulis [20, 21] and to research on symbiosis, it has clearly emerged that the boundaries between species are much more porous than imagined and that the possible interactions often involve contaminations, including also genetic ones [21, 22]. 


\section{Bats-Natural Host $\quad$ CoVs-Intermediate Hosts $\quad$ CoVs-Human Host}
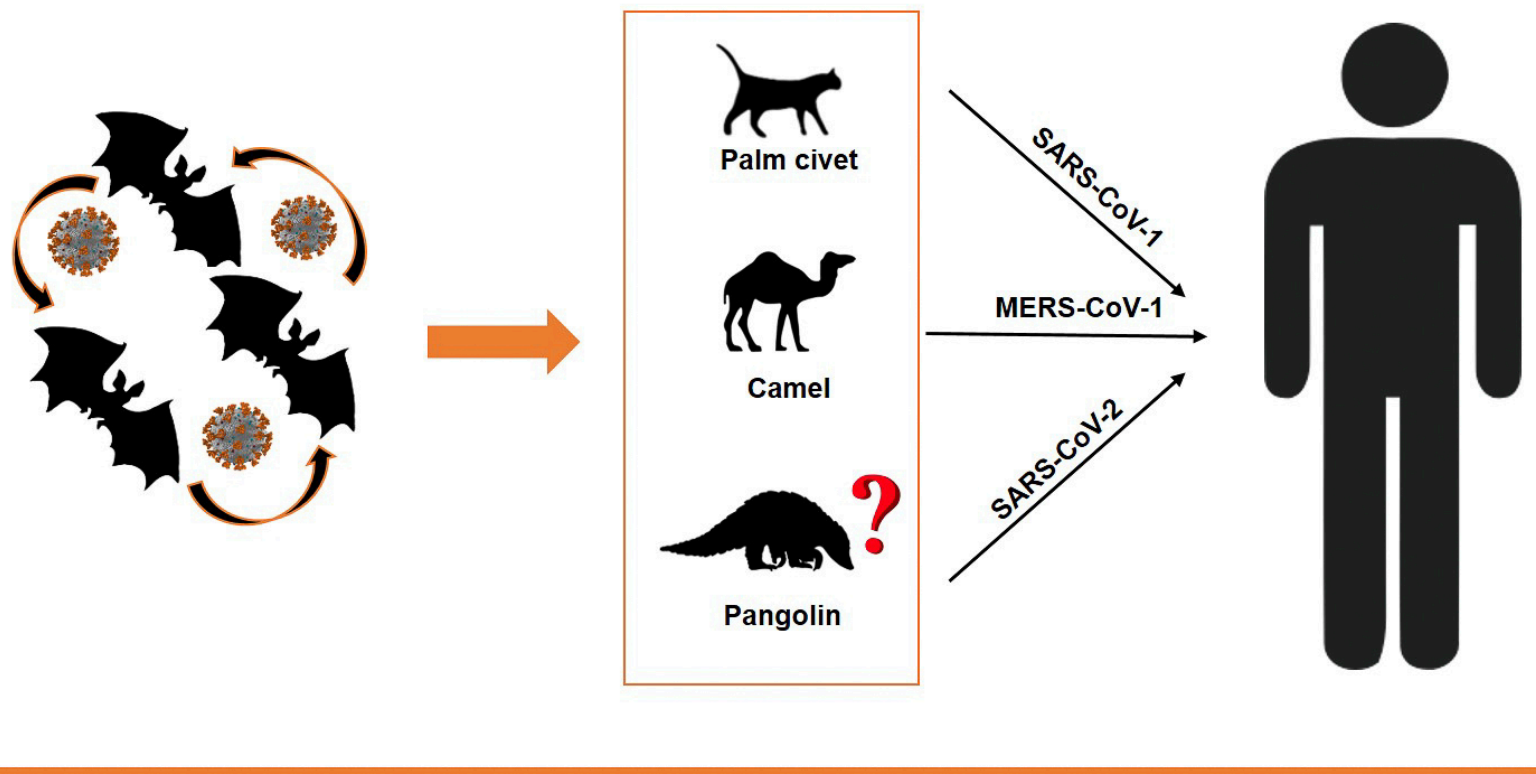

Fig. 1. Theorized animal origin of human coronaviruses. Bats are considered natural hosts while palm civet, camel and probably pangolin are intermediate hosts of different coronaviruses (CoVs). Human represents the final host.

Indeed, recent findings in molecular phylogenetics (the study of the evolutionary development of a species or a group of organisms or a particular characteristic of an organism) have revealed several novelties about our understanding of the relationship between the organisms that make up life on earth [15, 16, 22].

The phenomenon of horizontal gene transfer (HGT), which was initially thought to be isolated and circumscribed to bacteria and other microorganisms, is actually very widespread, concerning also animals and plants [23-26]. This has overturned the traditional view that inheritance only follows a vertical direction (from parents to offspring). Thus, this conception has shaped the way of understanding biological information and also the idea that this updated view on information, was a way to reconstruct the so-called tree of life. Evolution turned out to be more complex than previously thought. The famous metaphor of the tree is perhaps no longer so suitable to represent the life image, according to what the most recent data show. Evolutionary history is much less linear and much more intricate, as the type of biological information contained in genes is not only stored and reproduced within neatly traceable evolutionary trajectories. On the contrary, it can cross the barriers between species, genera, families, thus violating the boundaries of biological classification and taxonomy [16, 21-23, 25-29].
None of these new discoveries is just empirical. On the contrary, they also have a profound impact on our conceptions regarding the types of relations between species (even challenging them), including the extent of symbiotic processes and the nature of those phenomena defined as "infections".

On the one hand, in fact, new perspectives within symbiotic theory seem to suggest that the privileged organization units of the living are not macroscopic organisms (understood as those objects belonging to a specific species) but rather the ecological and biomolecular interaction networks that make up "functional assemblages" called "holobionts" [14, 27, 29]. This also explains how the genomes of organisms should also be considered in their interactive and relational stance, defined as "hologenome". An explicative holobiont example is that constituted by the human species and its associated microorganisms (i.e., the so-called microbiota).

The scope of these interactions is extremely profound. It can concern both aspects that govern the development of the so-called host (with consequences on the phenotype, including behavioral) and its regulatory framework (such as homeostatic processes). In this viewpoint, it is particularly crucial to mention the co-evolution of the immune system in relation to associated microorganisms. Moreover, other studies suggest that phylogenetics itself should be updated through the so-called "phylosymbiotic" 
perspective. In fact, phylosymbiosis is a new field of investigation that seeks to reconstruct the phylogeny of the host in relation to that of its associated microorganisms [16].

This relationship type is to be understood as, at least, bidirectional. On the one hand, the microbiota composition and its activities are associated with the development, maturation, and functionality of the immune system. On the other hand, the dynamics of the immune dimension are characterized starting from the relationship with the microbiota [11, 12, 30].

In this sense, the immune system must therefore be understood not only in its original meaning as a structure devoted to the host defense but also and above all as a global information arrangement of the organism, involved in the regulation of numerous systemic functions [31].

Moreover, even the genomes of each individual human being are no longer "pure", nor should they be intended in isolation but in turn, reveal an evolutionary story of interactions and contaminations. Not only has the human genome evolved in relation to its symbiotic microorganisms (and vice versa), but important portions of the human genome are made up of viral vestiges. From exogenous elements, these components have become integral parts of the genome, modifying its functionality and expressive possibilities [14, 15, 21, 22, 25, 26, 28, 29, 32, 33].

It is clear that all these developments, which lead to reconsidering the very nature of infections and the relationships between host, symbiotic organisms, and pathogens, pose challenges to the standard interpretation of the pandemic and its solutions.

\section{SARS-CoV-2 and the other causes of COVID-19 pandemic}

According to this perspective the new coronavirus known as SARS-CoV-2 is definitely responsible for the current COVID-19 pandemic, but it is not the only causal factor.

Coronaviruses constitute a sub-family of RNAviruses that may generate respiratory tract pathologies at least in mammals and birds [1, 18]. These viruses take advantage of a surface glycoprotein called "spike” which acts as a key for particular cell receptors (AngiotensinConverting Enzyme II or ACE2), thus invading cells and being replicated via the host's ribosome machinery $[1,7$, 18].

At the present state of knowledge, the transmission of the virus occurs mainly through respiratory droplets, which can be generated by coughing and sneezing but also by the activity of speaking or simply breathing if you are indoors without adequate air circulation $[1,18]$.

It is still unclear whether the spillover was direct (between humans and some species of bats) or if there is (as some suggest) an intermediate species [18]. This shows that the virus alone is not the cause of the pandemic but one cause. In order for the infection to become a global problem, and therefore not only medical/epidemiological but also socio-economic and political, it is necessary that there are other conditions and other determining causal factors.

In addition, in this case, the ecological dimension is central. On the one hand, the progressive destruction of the habitat of particular species of bats constitutes the primary access to the new infection. On the other hand, the presence of mixed markets (such as that of Wuhan) where wild meat is traded in urban areas, represents a further destabilization (because ecological barriers are reduced) and at the same time the creation of optimal conditions for the spread of a virus [7, 17].

In this viewpoint, therefore, if the proximate cause of the epidemic lies in the circulation of the pathogen and its mechanism of infection, the ultimate causes are to be found in the ecological dimension. This can mean at least two different (albeit related) lines of research.

On the one hand, in fact, studies in the field of ecology seem to suggest that the progressive alteration of global ecosystems constitutes a risk factor for the development of epidemics and pandemics. The reason is that while modifying ecosystems may eventually cause the extinction of certain species, while other species, particularly flexible (such as rodents and bats), can more easily become natural reservoirs of potential pathogens that are more likely to pass on to the human species [7, 9]. Therefore, the preservation of biodiversity and greater attention to these dynamics (e.g., policies to limit deforestation) are long-term measures that, acting on ultimate causal factors, could have a strong impact on future pandemics, greatly reducing their risk $[9,17]$. So, we also involve the adoption of different approaches (corresponding to diverse lines of research) within the ecology itself. On the one hand, in fact, ecology is intertwined with epidemiology as regards population studies on the transmission of diseases and the environmental conditions (including biotic and abiotic factors) that influence these mechanisms. In addition, this process has an impact on the modeling of global phenomena such as a pandemic.

In conclusion, ecology is also a precious and indispensable source of studies concerning patho-symbiotic interactions, also placing them in different biogeographic contexts. To this must be linked those experimental ecology studies that analyze the spread and development of pathologies in relation to biodiversity [34].

The other branch of the ecological dimension is represented by the need to pay attention to biodiversity. In particular, symbiotic functional units (such as the holobiont) need, for the purposes of general health, a balance of specific determinants of environmental biodiversity (both internal and external). For example, human urbanization had a significant impact on microbial diversity, basically reducing it and eliminating numerous opportunities for interaction with exchanges from other environmental contexts. 
Indeed, a highly urbanized life in countries with a high rate of industrial development is associated with some specific lifestyles (e.g., sedentary), exposure to different environmental pollutants, but also better health conditions (public hygiene, antibiotics, vaccination). As a matter of fact, all of these elements have the potential to increase risk factors for infectious diseases such as COVID-19 [35]. However, here we mean "internal" biodiversity, that of the microbiota. Obviously, the microbiota composition also depends on external factors such as diet and lifestyles, as well as on the context conditions in which the host lives. However, the ecological perspective of interest here concerns the specific systemic dimension of the microbiota in relation to pathogens and the immune system.

Thus, future treatments for viral infections and the pandemic could benefit from a greater understanding of how the microbiota interacts with these two elements. In addition, a rather recent field of studies regarding this perspective, sees the range of different approaches: from microbial diversity to exposomics (i.e., the analysis of the totality of agents to which the organism is exposed), up to ecology restoration. In this view, some researchers have proposed the possibility of developing microbiomeinspired green infrastructure (MIGI). These achievements involve the dynamic restructuring of the living environment taking into account both macro-biodiversity (external) and micro-biodiversity (internal) [7, 10, 36-43].

\section{Viruses and pandemics from an ecological standpoint}

Viruses are everywhere and interact with every known life form. Their nature, however, is debated. Some argue that they should be conceived as "living beings" while others reject this hypothesis [44]. The question is almost more philosophical than scientific. However, it is ascertained that viruses play a fundamental role in the dynamics concerning the genesis and development of all living forms $[18,44]$. There are still many difficulties regarding the taxonomy and classification of viruses, their phylogeny, but given their pervasiveness, there are also those who have hypothesized that they are one of the determining factors of the evolutionary process $[16,44]$.

As previously reported, epidemics and pandemics are therefore not new in the history of the human species. Infectious diseases have not only contributed to determining the development of the human species (operating as a selective filter) but have also conditioned its fate by virtue of the fact that they offer a perspective on the human interaction with other living forms [16, 22, 44].

Precisely for this reason, it is crucial to note how the globalization phenomena and the massive modification of ecosystems by the human species are two essential factors for understanding the pandemic known as COVID-19. In this sense, several aspects must be better specified.
In the first place, the social and technological modality with which the human species has configured itself in the last century constitutes a particularly suitable framework for the spread of pandemics. Contemporary human societies are made up of millions (sometimes billions) of individuals who are concentrated above all in certain areas where they live in close contact according to dynamics that provide for strong social and physical interaction.

Second, as previously reported, the impact of human activities on global ecosystems has been increasingly profound and very often with disruptive and destructive outcomes. This not only changed the existence and behavior of other animal and plant species but also affected microorganism ones, thus generating new possibilities for contact and interaction with several potential pathogens.

It is, therefore, no mystery that the interactions between infectious agents, their hosts, and the environment show how the cause of pandemics cannot be reduced exclusively to a medical and epidemiological problem. While it is certainly true that the presence of pathogens, and their characteristics, is a necessary condition to be able to talk about epidemics/pandemics, it is not sufficient. The characteristics of the species involved, that is the infection object, are equally crucial, as is the environmental context. This context does not only concern the so-called "natural” environment (defined in such a simplified manner) but also the general context conditions in which all organisms live. Furthermore, the environment is not simply a container for the species, but a set of biotic and abiotic factors that influence the development of the species and are in turn modified by it, in a sort of dialectical relationship [10]. In this perspective, it is important to remember how several studies have highlighted the high impact of COVID-19 on both human and animal health and behavior. First of all, this is true not only because of the infection itself but also by measuring the consequences on humans of the measures designed to combat the pandemic. These measures not only had an effect on the mental health and social well-being of human beings but also had a cascading impact on all human activities. As a result, mediated effects have also been recorded on other species and environments. These can be both positive (such as greater attention to biodiversity protection and the imposition of restrictions on the consumption of wild species) and negative (such as the production of waste resulting from the implementation of health regulations to stop the virus spread) [45]. Furthermore, other research has shown how lockdown measures and the slowdown of certain economic activities have had a positive impact, in some respects, on environmental sustainability. Given that this situation is destined to change, with a view to a return to so-called "normal" conditions, it is appropriate to evaluate not only how to draw inspiration from the (economic and ecological) constraints imposed by the pandemic but also how viruses react and are shaped by such countermeasures [46]. 
This ecological dimension is crucial when the genetic-molecular characteristics of infectious agents, such as viruses, are explored.

For example, viruses (especially RNA viruses) show great plasticity and genomic instability that leads them to be able to change with extreme speed through repeated mutations. This allows viruses to be particularly capable of adapting to new conditions, responding to changes (even drastic changes in context), or successfully modifying some mechanisms of the infection itself [19, 44].

This can result in changes in the infectious capacity, the selectivity towards certain species (as the human one), or the different danger towards particular categories of individuals (e.g., the elderly ones) [18, 19].

Obviously, the characteristics of the infected species are also fundamental. This involves understanding which receptors might guarantee or favor infection, on which cells these receptors are most expressed, and what consequences this may have for the organism. It is clear that the greater or lesser susceptibility of some cell types and of specific tissues, which are of systemic importance, has a crucial impact on the disease genesis and development for the whole organism. Additionally, there may be human genetic variants that may or may not favor viral infection $[7,18,19]$. Finally, the behavior of the species, as already mentioned, constitutes another crucial factor. In fact, contemporary human societies, especially those of highly industrialized countries, determine some ideal conditions for a virus that spreads by air, through saliva particles [9, 17].

\section{Infections and microbiota}

The role of microbiota in the host's health status has been proven to be crucial. In this perspective, that means not only that microbiota is directly involved in the functionality of the immune system as such $[11-13,30]$ but also that it somehow influences pathogens' activities. Indeed, microorganisms can shape pathogens' capability to onset an infection through the so-called colonization resistance theory (according to which commensal bacteria can compete with pathogens for specific niche occupation, thus helping the host against infections). Moreover, it is now widely recognized that microbiota contributes to immune cells training, differentiation, and specific functionality [11, 12, 14, 42, 47].

Furthermore, commensal microorganisms play a role in secondary lymphoid tissue activity [38]. In addition, gut inflammation or indiscriminate antibiotics' use may generate alterations in microbiota composition, thus favoring pathogens' activities. On the contrary, specific therapeutic interventions which display a distinctive ecological stance, such as fecal microbiota transplantation (FMT), have shown to be very effective in contrasting antibiotic-resistant bacteria such as Enterococcus faecium, gram-negative Enterobacteriaceae and Clostridium diffi- cile [14, 47]. Moreover, some studies suggest that probiotics, by acting on microbiota (again in an ecological frame), can enhance the immune response or simply diminish the chances for pathogens to establish infections [37].

In addition, these features suggest how microbiota can interact with viruses. Indeed, commensal microorganisms can affect viral infections, either promoting or inhibiting viral activities and functionality, in several ways. In detail, recent studies show how specific bacterial strains can influence viral fitness, or how synergistic effects can be created due to the alteration of prokaryotic metabolism by viruses, pushing bacteria to change their mode of interaction with the host, switching from commensal to pathogenic behavior [48]. Indeed, it should be noted that the ability to infect the host by viral agents also depends on some context factors (such as the protective characteristics of the epithelial cells or the $\mathrm{pH}$ of the environment) which can be greatly influenced by microbial activity [13, 36, 38-41, 43, 48-50]. Moreover, in this case as well, the bidirectional crosstalk with the immune system can definitely play a role in shaping the response and the outcome of infections of viral origin. As a matter of fact, the relationship between viral agents and microbiota, regarding the potential interaction, is almost obvious given that the crossing of the epithelial cells of the mucosa remains an obligatory step.

Indeed, by considering that the human microbiota does not reside only in the intestine but colonizes various body areas (especially where there are strong interactions with the outside, e.g., lungs and respiratory tract, genitals, and oral cavities) it is almost certain that viral infections have an impact and are modified by microbial activity (both indirectly and directly). Studies with germ-free animal models have offered new clues to these interactions and potential therapeutic interventions [41, 42]. Finally, it should be remembered that critical clinical conditions and deaths from COVID-19 are essentially associated with comorbidities which result, in the majority of cases, in marked (and often dysfunctional) alterations of the patients' microbiota. Notably, these critical clinical situations often require complex therapeutic interventions (and based on drugs' combinations), which can have a severe impact on the gut microbiota composition and functionality. Disregarding this dimension could lead to a worsening of clinical conditions (among other things not foreseen by traditional protocols). Therefore, including the microbiota in the clinical evaluation, testing the impact of drugs on its composition and functionality necessarily implies also a greater understanding of the contribution of microorganisms to global health and therefore requires the development of both integrated and specific approaches (and which could also act as a trailblazer for future forms of therapeutic intervention in general) [51]. 


\section{COVID-19 and microbiota}

Although SARS-CoV-2 transmission occurs mostly through respiratory droplets, growing evidence seems to suggest a possible, not neglectable interaction with the microbiota.

As mentioned, the microbiota manipulation can also take place through interventions of an ecological nature, aimed at shaping the composition and functionality of the microbiota itself, with effects on the general host health [14, 32].

In fact, some interactions between the host and microbiota (a situation sometimes called “eubiosis") show certain characteristics that are extremely important in the case of infectious diseases. For example, diets rich in carbohydrates or high-fat not only can alter, via gut microbiota, some global biological functions (such as the sleep-wake cycle) that have homeostatic importance for the host health but also favor (due to the interaction of commensal microbes with the immune system) an increased condition of sustained inflammation that can make the individual more susceptible to external infections [13, 14]. Recent studies have clearly shown how the status and composition of the microbiota play a non-trivial role in shaping the host immune response to COVID-19 infection and in determining any comorbidities [52].

Conversely, different studies show how the intake of vegetable proteins and a low-fat diet not only increase the presence of some bacteria genera, such as Bifidobacterium and Lactobacillus, which are associated with general well-being of human health, but they also favor these genera at the expense of potential pathogens such as Bacteroides fragilis and Clostridium perfringens [13].

Indeed, other studies have shown that plant fibers and other prebiotic compounds are associated with better body function (e.g., better immune response and better digestive activities) also reducing insulin resistance or decreasing the general level of given cytokines. These outcomes are crucially associated with some metabolic activities of the commensal bacteria (such as the production of short-chain fatty acids or SCFAs) which seem to influence not only the local immunity, i.e., of the intestinal tract, but also the global one and, more interesting for the COVID-19 pandemic, the respiratory tract [7, 13, 38].

In addition to this, it is also worth remembering the importance of the lung microbiota. Having received less attention than the intestinal one, the lung microbiota is not yet well characterized (both because the routes of entry and direct colonization are still poorly studied and because there are cases of bacterial translocation from the colon to the lungs). Nevertheless, a very recent review has highlighted how a deeper knowledge of the differences in the composition of the lung microbiota can be crucial to unravel determining factors for the onset of acute respiratory distress syndrome (ARDS) linked to COVID-19 infection
[53]. Moreover, some genera (such as Prevotella and Veillonella) are correlated with different respiratory diseases (it is still not clear to what extent positively or negatively). Recent studies have shown how specific metabolic products of lung bacteria can have a synergistic effect on the infection severity [54]. Even in this case, although a mechanistic understanding of the phenomenon is still lacking, colonization resistance theory provides some clues as to how certain bacterial populations can offer an additional deterrent to pathogen infections [38, 53, 55].

Summing up, we can claim that, generally speaking, greater biodiversity of the microbiota can crucially contribute to the general well-being of the individual and to his health status, thus making him more capable of resisting infections by exogenous pathogens.

Another interesting aspect of the potential intersections of microbiota research with the ongoing pandemic concerns the comorbidities between COVID-19 and other conditions.

In fact, recent studies have shown that, during the coronavirus infection phase, there is a high incidence (around $40 \%$ of patients) of intestinal disorders such as vomiting or diarrhea [53, 55]. In addition, and considering a more ecological point of view, it should be remembered that numerous detection studies of the SARS-CoV-2 virus on surfaces in hospital environments (or in any case related to the context in which the patient is) can be crossed with the presence of specific bacterial presence. In particular, some recent data have documented how bacterial community profiles allowed a more efficient accuracy for the prediction of SARS-CoV-2 presence [56]. These gut disruptions also increase the possibility that patients will have to undergo more stringent measures and treatments, such as intensive care, with a formidable impact on the resilience of health systems. Obviously, this does not mean that these symptoms are a direct consequence of the viral infection but they could occur as side effects or could be promoted by specific comorbidities [38, 41, 55]. Some recent observational studies have also shown how COVID-19 patients present a decrease in microbial diversity and an increase of some bacterial genera (favoring those which produce less SCFAs) compared to healthy control groups [36, 41, 55]. Furthermore, very recent results suggested that the complex relationship between diet, microbiota activity, and infection may constitute a neglected aspect of the infectious capacity and the host response to SARS-CoV-2 infection. Given the danger of decreasing microbial biodiversity in COVID-19 patients, mainly through antibiotics, a more detailed understanding of the bacterial strains involved in these processes could lead to the development of more targeted strategies, perhaps thanks to the use of nanotechnologies (both for delivery of specific nutrients in the gut and to selectively affect only relevant strains) [57]. 
Furthermore, it cannot be excluded that the infection can also be transmitted via the fecal-oral route. In fact, more than half of infected patients show the presence of the virus in the feces and asymptomatic patients also showed the virus presence with a charge capable of causing infection $[41,55]$. Interestingly, the receptor (ACE2) to which the virus binds, present in the epithelial cells of the lungs, is expressed by many other cell types, including those of the intestinal epithelium. In this scenario, it has been shown how angiotensin-converting enzyme 2 (ACE2) receptor is associated with intestinal immune response and, even more strikingly, with gut microbial ecology, affecting both its composition and functionality [18, 41, 55]. The potential crosstalk between microbiota activities and ACE2 expression and regulation, in association with specific unfavorable outcomes in COVID-19 patients, suggests that this system could become a novel target for potential and promising therapeutic strategies [36, 41, 50, 55].

Another aspect concerns the comorbidities that present an altered picture also from the point of microbiota view. First of all, obesity (also due to the difficulties that this condition entails for the cardio-respiratory system) is usually associated with a higher and more constant inflammatory response. Moreover, it has been noticed that the adipocytes of obese patients show an overexpression of the ACE2 receptor [38, 55]. Type II diabetes and hypertension are also conditions associated with global alterations of the immune response and more recently with dysbiosis and other forms of microbiota modifications [38, 49].

The other very important data concerns the microbiota variation with aging. It is known that the microbiota variability decreases in elderly subjects and that the composition of the microbial ecosystem changes with age. This variation is most often associated with a decrease in some systemic functions (including an increase in inflammatory markers). Given that the vast majority of deaths from COVID-19 concern the elderly population, it cannot, therefore, be excluded that a reduced functionality of microbiota may contribute as an aggravating prognostic factor to the outcome of the infection. For this reason, forms of therapeutic and, above all, preventive measures and integration towards elderly patients, in the future could concern nutrition and therefore the microbiota shaping [18, 38].

Interestingly, recent studies have shown how the microbiota can modulate the function of the immune system in relation to vaccination. Clinical and preclinical studies have shown how the microbiota composition has an impact on the immune response [39, 40, 58, 59]. In fact, starting from in vivo studies on animal models (both germ-free and antibiotic-treated specimens) it was found that the antibody responses to the influenza vaccine were altered, thus suggesting that the presence of commensal microorganisms may also have a function in determining the vaccine efficacy $[39,58]$. In humans, a positive correlation has been found between the presence of the phylum Actinobacteria and the response to some vaccinations such as hepatitis B and poliomyelitis, while the Enterobacteriaceae family appears to be negatively correlated [38].

Unfortunately, a detailed and complete mechanistic understanding of how the microbiota interacts with the immune response has not been achieved yet and a direct link between commensal functionality and vaccine efficacy cannot be established. However, in recent times, different studies show how eubiosis (i.e., the situation in which the microbiota and its metabolic activity positively contribute to the host functionality) is definitely related to a higher variety of disposable pathways, regulating the immune response [13, 38].

Also, from this point of view, the ecological dimension (so far neglected) seems to show its importance. In fact, both the immune response and the pathogen efficacy, as well as the diversity and richness of the microbiota also depend, alongside internal factors (such as genetic ones), on environmental factors. Elements such as type of diet, climate, geography, lifestyles, cultural habits, and procedures can influence both the severity of the infection and the success of a therapeutic intervention. So far, wastewater surveillance has been proposed as a powerful tool for monitoring factors related to human health such as infectious diseases, in particular, to find out the efficacy of public health interventions. In light of this since the beginning of the pandemic, surveillance of wastewater samples has been regarded as a possible and promising approach to monitor the SARS-CoV-2 circulation [60, 61]. Understanding these factors better and trying to assess their weight constitutes a challenge that cannot be postponed with a view to a future fight against pandemics. In fact, a body of evidence is growing concerning unsatisfactory responses to vaccines in so-called "developing” countries. This scenario suggests how medicine is urgently called upon to incorporate, in its clinical frame; elements once considered external but which increasingly show to be crucial determinants for health $[14,17]$.

Last but not least, the troubles in having a complete and clear picture of this situation clearly show the difficulties of reducing the understanding of certain phenomena to a mainly mechanistic description and instead reiterate the need for an ecological perspective that goes beyond current practices of biomedical research. This means also to recognize that, in considering the connections between microbiota and coronavirus, we are not only facing with single interactions but rather with a complex network that includes different actors, such as the host and associated microorganisms (i.e., the "holobiont”), pathogens (including viruses) and the various contextual conditions. Only if we import models and methodologies to deal with this type of scenario, the providing of an explanatory framework (which allows the experimental manipulability of these phenomena and therefore on the possible therapeutic solutions) will be eventually possible [8, 10, 14] (Fig. 2). 


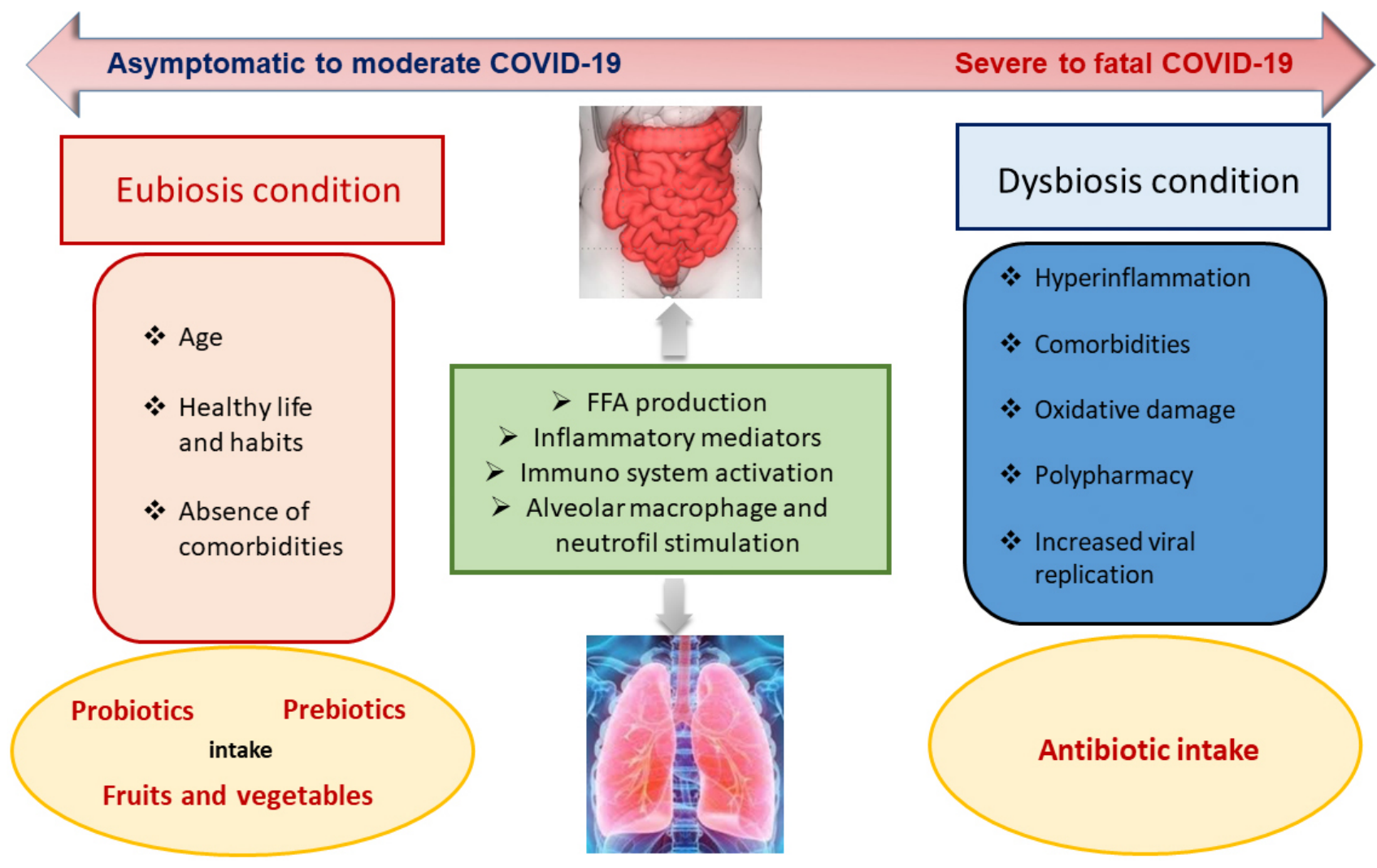

Fig. 2. Representative picture of the impact of eubiosis vs. dysbiosis on the outcome of COVID-19 patients. The elaborate crosstalk between the gut-lung axis could be crucial in influencing the susceptibility of airways to SARS-CoV-2 infection and subsequent clinical outcome as a result of the inter-individual differences in composition and function of microbiota.

\section{Ecological perspective in medicine and future directions}

Understandably, considering emergency and urgency, the first forms of fighting the virus and the pandemics were physical distancing and sanitation protocols.

However, a recent perspective proposes a critical analysis of the very concept of "hygiene" in the context of modern health systems [7, 17]. Indeed, if it is true that the development of public health (especially in Western countries) and the very promotion of the concept of "hygiene" have eradicated many infectious diseases and considerably increased people's life expectancy, it is equally true that this model, typical of a progressively urbanized society, constituted of increasingly mediated interactions, has also produced a significant decrease in the "beneficial" microbial populations and also in the biodiversity of the human microbiota [7, 17].

This theoretical framework proposes that the changes, over time, which various human populations have undergone, have determined a consistent loss of microorganism biodiversity. These changes concern some characteristic elements of contemporary life (especially in Western countries): urbanization, indiscriminate use of antibiotics, the sanitation of living and working environments, the homologation of food (towards a greater presence of industrially produced foods), and excessive consumption of alcohol and tobacco [17]. This diversity loss is associated with the development of specific diseases, such as autoimmune ones, but also has a link with susceptibility to some infectious diseases [7, 17]. These are very often conditions that are difficult to manage, in the current state of research, given that recovering this biodiversity loss is particularly challenging, as the studies in ecology, concerning the extinction and reduction of the number of species, clearly show $[6,9]$.

Currently, sanitation, together with physical distancing and vaccines, is a tool that can hardly be replaced. However, the tightening of some hygiene measures in the most varied contexts also constitutes a threat to microbial diversity that risks being further reduced. In the future, there are some researchers who believe it is necessary to rethink, extend and adapt the theoretical-practical assumptions related to the hygiene concept to make it more functional to fight pathogens but at the same time not destructive towards microorganism biodiversity $[9,17]$. In order to achieve this, that is to develop selective models and approaches, which are capable of containing infectious agents but which at the same time do not destroy beneficial microorganisms (which could also constitute a barrier in themselves towards their pathogenic counterpart), there is ur- 
gency for an ecological perspective to be imported into the biomedical sciences [14].

The COVID-19 pandemic seems to have more clearly shown that some key factors (including those concerning measures to contain the virus spread) such as the loss of biodiversity, socio-economic inequalities, and the different possibilities of access to specific health resources, are as central as the mechanism of viral infection, if not more than it, in explaining the current situation. This is because, if we want to answer the question about why the pandemic occurred, perhaps the responsibility is not directly of the virus but of the organization of health systems (especially in many western countries) and of the global socioeconomic development model [17].

There is no doubt that this COVID-19 pandemic has been able to hit the currently dominant model of society and economy in a disruptive way and at a level, which has very few precedents in recent history. The inability to react promptly to this phenomenon, the misplaced security in the efficiency of the Western systems (defined as "best health systems in the world"), have been clearly visible during 2020. To date, the situation cannot be said to be over. Luckily, the vaccines' development (along with other pharmacological treatments that might be developed in the future) would be able to guarantee the end of the acute phase of the pandemic but it will not solve what is at the phenomenon root, i.e., its ultimate causes. If these causes are not paid attention to, it is not so unlikely that humanity will soon find itself facing such an emergency again with the risk of finding itself unprepared again.

In the particular case of Western countries, the pandemic has shown all the criticalities not only of the health model but also of the ideas of economic growth and development [17].

This translates into taking into consideration the pandemic in its broader meaning for which it is not just a medical-health problem.

First of all, this means investing in research that can shed light on the dynamics with which zoonoses are possible, not only from the point of view of the spillover mechanism but also and above all what are the ecosystemic and socio-economic factors that make them more probable and more serious. For example, it is now clear that intensive farming, large market centers increase the likelihood of outbreaks, and that the selective pressures of such contexts can aggravate the infectious capacity of the pathogens. Furthermore, these farms usually provide for the use of broadspectrum antibiotics on livestock that not only impacts the animal microbiota but in turn translate into a reduced ability to enrich the human one. These factors must also be inserted into broader issues concerning the footprint of the human species on the planet (such as the climate crisis of anthropogenic origin), which have been shown to have profound consequences on almost all life aspects on earth and in particular, concerning the food production dimension and human lifestyle. Unfortunately, these aspects seem to give an unfavorable direction towards the microbial richness that is so essential for human health $[9,14,17]$.

\section{Conclusions}

The COVID-19 pandemic has caught the world off guard. If on the one hand, the responses to this health emergency have focused on health aspects, it seems necessary to broaden both the gaze of understanding and the space for intervention, to avoid making the same mistakes in the future.

On the one hand, this is due to changes of a theoretical nature. Current ideas about diseases, infections, what constitutes a pathogen, are in a phase of progressive revision due to the ever-increasing research on the symbiotic and ecosystem dimension, which shows how life forms on earth are much more connected and in the relationship between them than previously believed.

Indeed, we have begun to understand the role of viruses in the evolutionary framework and now it is clear that the existence of our species is intimately linked to that of the associated microorganisms that populate our body: the microbiota. It is now known that the microbiota has a crucial impact on an ever-increasing number of activities that affect and determine the host functionality, from the immune system (including perhaps susceptibility to vaccines) to general homeostasis, up to the cognitive dimension [11, 12, 27, 32].

A greater understanding of these aspects necessarily requires broadening the current perspective in biomedicine about concepts such as "prevention”, “causal factors of diseases" and "hygiene", towards a theoretical framework that involves, more markedly, the ecological dimension. Thus, clinical models that do not yet include these factors in establishing what health is, and what it means to be sick are increasingly at risk of being incomplete.

A growing group of experts believes that future responses to the pandemic can only benefit from these extensions. Furthermore, the studies on the microbiota, which arise from the encounter of medicine with microbial ecology and in the wake of an evolutionary perspective, also constitute a fruitful field of investigation to include, in an operational way, some determinants of health that have been traditionally poorly considered (or even neglected) by doctors (such as socio-economic factors) [10, 14].

Further development of microbiota studies could link these factors with greater precision and clinical relevance. Therefore, the hope is that the medicine of the future could have both the ability to analyze and the disposal of preventive measures also on aspects that nowadays concern other investigation fields. With these tools, perhaps, medicine will also be able to deal with the ultimate causes of epidemics and not just the proximate ones. 


\section{Author contributions}

Conceptualization and design of the study, all the authors; writing-original draft preparation, FB; figures and bibliographic revision, GN, critical revision of the article, all the authors; final approval of the version of the paper: all the authors; supervision, AA.

\section{Ethics approval and consent to participate}

Not applicable.

\section{Acknowledgment}

We thank Leandro Di Gloria for his support in revising the bibliography.

\section{Funding}

This study received no external fundings.

\section{Conflict of interest}

The authors declare no conflict of interest. Amedeo Amedei is serving as one of the Editorial Board members of this journal. We declare that AA had no involvement in the peer review of this article and has no access to information regarding its peer review. Full responsibility for the editorial process for this article was delegated to GP.

\section{References}

[1] Morens DM, Fauci AS. Emerging Pandemic Diseases: how we Got to COVID-19. Cell. 2020; 183: 837.

[2] Dror AA, Eisenbach N, Taiber S, Morozov NG, Mizrachi M, Zigron A, Srouji S, Sela E. Vaccine hesitancy: the next challenge in the fight against COVID-19. European Journal of Epidemiology. 2020; 35: 775-779.

[3] MacDonald NE. Vaccine hesitancy: Definition, scope and determinants. Vaccine. 2015; 33: 4161-4164.

[4] Sarewitz D. Slow science. Issues in science and technology. Fast science. 2020; 36: 18-19.

[5] MAYR E. Cause and effect in biology. Science. 1961; 134: 1501-1506.

[6] Aguirre AA. Changing Patterns of Emerging Zoonotic Diseases in Wildlife, Domestic Animals, and Humans Linked to Biodiversity Loss and Globalization. ILAR Journal. 2017; 58: 315-318.

[7] Finlay BB, Amato KR, Azad M, Blaser MJ, Bosch TCG, Chu H, et al. The hygiene hypothesis, the COVID pandemic, and consequences for the human microbiome. Proceedings of the National Academy of Sciences. 2021; 118: e2010217118.

[8] Buck JC, Weinstein SB. The ecological consequences of a pandemic. Biology Letters. 2020; 16: 20200641.

[9] Gibb R, Redding DW, Chin KQ, Donnelly CA, Blackburn TM, Newbold T, et al. Zoonotic host diversity increases in humandominated ecosystems. Nature. 2020; 584: 398-402.

[10] Roche B, Garchitorena A, Guégan J, Arnal A, Roiz D, Morand $\mathrm{S}$, et al. Was the COVID-19 pandemic avoidable? A call for a "solution-oriented" approach in pathogen evolutionary ecology to prevent future outbreaks. Ecology Letters. 2020; 23: 15571560.

[11] Belkaid Y, Hand TW. Role of the microbiota in immunity and inflammation. Cell. 2014; 157: 121-141.

[12] Belkaid Y, Harrison OJ. Homeostatic Immunity and the Microbiota. Immunity. 2017; 46: 562-576.

[13] Ruff WE, Greiling TM, Kriegel MA. Host-microbiota interactions in immune-mediated diseases. Nature Reviews Microbiology. 2020; 18: 521-538.

[14] Ronai I, Greslehner GP, Boem F, Carlisle J, Stencel A, Suárez J, et al. "Microbiota, symbiosis and individuality summer school" meeting report. Microbiome. 2020; 8: 117.

[15] Rook G, Bäckhed F, Levin BR, McFall-Ngai MJ, McLean AR. Evolution, human-microbe interactions, and life history plasticity. Lancet. 2017; 390: 521-530.

[16] Lim SJ, Bordenstein SR. An introduction to phylosymbiosis. Proceedings of the Royal Society B: Biological Sciences. 2020; 287: 20192900.

[17] Leach M, MacGregor H, Scoones I, Wilkinson A. Postpandemic transformations: how and why COVID-19 requires us to rethink development. World Development. 2021; 138: 105233.

[18] Sironi M, Hasnain SE, Rosenthal B, Phan T, Luciani F, Shaw M, et al. SARS-CoV-2 and COVID-19: a genetic, epidemiological, and evolutionary perspective. Infection, Genetics and Evolution. 2020; 84: 104384.

[19] Vos M. The evolution of bacterial pathogens in the Anthropocene. Infection, Genetics and Evolution. 2020; 86: 104611.

[20] Corliss JO, Margulis L. Origin of Eukaryotic Cells. Transactions of the American Microscopical Society. 1972; 91: 442.

[21] Guerrero R, Margulis L, Berlanga M. Symbiogenesis: the holobiont as a unit of evolution. International Microbiology. 2013; 16: 133-143.

[22] Gilbert SF, Sapp J, Tauber AI. A Symbiotic View of Life: we have never been Individuals. The Quarterly Review of Biology. 2012; 87: 325-341.

[23] Chen R, Huangfu L, Lu Y, Fang H, Xu Y, Li P, et al. Adaptive innovation of green plants by horizontal gene transfer. Biotechnology Advances. 2021; 46: 107671.

[24] Rodríguez-Beltrán J, DelaFuente J, León-Sampedro R, MacLean RC, San Millán. Beyond horizontal gene transfer: the role of plasmids in bacterial evolution. Nature Reviews Microbiology. 2021; 19: 347-359.

[25] Sibbald SJ, Eme L, Archibald JM, Roger AJ. Lateral Gene Transfer Mechanisms and Pan-genomes in Eukaryotes. Trends in Parasitology. 2020; 36: 927-941.

[26] Van Etten J, Bhattacharya D. Horizontal Gene Transfer in Eukaryotes: not if, but how much? Trends in Genetics. 2020; 36: 915-925.

[27] Bordenstein SR, Theis KR. Host Biology in Light of the Microbiome: Ten Principles of Holobionts and Hologenomes. PLoS Biology. 2015; 13: e1002226.

[28] Gabaldón T. Patterns and impacts of nonvertical evolution in eukaryotes: a paradigm shift. Annals of the New York Academy of Sciences. 2020; 1476: 78-92.

[29] Rees T, Bosch T, Douglas AE. How the microbiome challenges our concept of self. PLoS Biology. 2018; 16: e2005358.

[30] Eberl G. A new vision of immunity: homeostasis of the superorganism. Mucosal Immunology. 2010; 3: 450-460.

[31] Pradeu T, Jaeger S, Vivier E. The speed of change: towards a discontinuity theory of immunity? Nature Reviews Immunology. 2013; 13: 764-769.

[32] Amedei A, Boem F. I've gut a feeling: Microbiota impacting the conceptual and experimental perspectives of personalized medicine. International Journal of Molecular Sciences. 2018; 19: 3756.

[33] Fambrini M, Usai G, Vangelisti A, Mascagni F, Pugliesi C. The plastic genome: the impact of transposable elements on gene 
functionality and genomic structural variations. Genesis. 2020; 58: e23399.

[34] Hurst C. The Connections Between Ecology and Infectious Disease. Springer . 2019.

[35] Mills JG, Brookes JD, Gellie NJC, Liddicoat C, Lowe AJ, Sydnor HR, et al. Relating Urban Biodiversity to Human Health with the 'Holobiont' Concept. Frontiers in Microbiology. 2019; 10: 550 .

[36] Baghbani T, Nikzad H, Azadbakht J, Izadpanah F, Haddad Kashani H. Dual and mutual interaction between microbiota and viral infections: a possible treat for COVID-19. Microbial Cell Factories. 2020; 19: 217.

[37] Baud D, Dimopoulou Agri V, Gibson GR, Reid G, Giannoni E. Using Probiotics to Flatten the Curve of Coronavirus Disease COVID-2019 Pandemic. Front Public Health. 2020; 8: 186.

[38] Dhar D, Mohanty A. Gut microbiota and COVID-19- possible link and implications. Virus Research. 2020; 285: 198018.

[39] Kim Y. Microbiota Influences Vaccine and Mucosal Adjuvant Efficacy. Immune Network. 2017; 17: 20.

[40] Dhakal S, Klein SL. Host Factors Impact Vaccine Efficacy: Implications for Seasonal and Universal Influenza Vaccine Programs. Journal of Virology. 2019; 93: e00797-19.

[41] Vodnar DC, Mitrea L, Teleky BE, Szabo K, Călinoiu LF, Nemes SA, Martău GA. Coronavirus Disease (COVID-19) Caused by (SARS-CoV-2) Infections: A Real Challenge for Human Gut Microbiota. Frontiers in Cellular and Infection Microbiology. 2020; 10: 575559.

[42] Watkins A, Bocchi F. Pathogen versus microbiome causation in the holobiont. Biology \& Philosophy. 2019; 35: 7.

[43] Mills JG, Brookes JD, Gellie NJC, Liddicoat C, Lowe AJ, Sydnor HR, et al. Relating Urban Biodiversity to Human Health with the 'Holobiont' Concept. Frontiers in Microbiology. 2019; 10: 550 .

[44] Harris HMB, Hill C. A Place for Viruses on the Tree of Life. Frontiers in Microbiology. 2021; 14: 604048.

[45] Buck JC, Weinstein SB. The ecological consequences of a pandemic. Biology Letters. 2020; 16: 20200641.

[46] Saleh J, Peyssonnaux C, Singh KK, Edeas M. Mitochondria and microbiota dysfunction in COVID-19 pathogenesis. Mitochondrion. 2020; 54: 1-7.

[47] Vangoitsenhoven R, Cresci GAM. Role of Microbiome and Antibiotics in Autoimmune Diseases. Nutrition in Clinical Practice. 2020; 35: 406-416.

[48] Neu U, Mainou BA. Virus interactions with bacteria: Partners in the infectious dance. PLoS Pathogens. 2020; 16: e1008234.

[49] Rume T, Islam SMD. Environmental effects of COVID-19 pandemic and potential strategies of sustainability. Heliyon. 2020; 6: e04965.

[50] Villena J, Kitazawa H. The Modulation of Mucosal Antiviral Immunity by Immunobiotics: Could They Offer Any Benefit in the SARS-CoV-2 Pandemic? Frontiers in Physiology. 2020; 11: 699.
[51] Donati Zeppa S, Agostini D, Piccoli G, Stocchi V, Sestili P. Gut Microbiota Status in COVID-19: An Unrecognized Player? Frontiers in Cellular and Infection Microbiology. 2020; 10 576551.

[52] Yeoh YK, Zuo T, Lui GC, Zhang F, Liu Q, Li AY, et al. Gut microbiota composition reflects disease severity and dysfunctional immune responses in patients with COVID-19. Gut. 2021; 70 698-706.

[53] He Y, Wang J, Li F, Shi Y. Main Clinical Features of COVID19 and Potential Prognostic and Therapeutic Value of the Microbiota in SARS-CoV-2 Infections. Frontiers in Microbiology. 2020; 11: 1302.

[54] Dragelj J, Mroginski MA, Ebrahimi KH. Hidden in Plain Sight Natural Products of Commensal Microbiota as an Environmental Selection Pressure for the Rise of New Variants of SARSCoV-2. ChemBioChem. 2021; 22: 2946-2950.

[55] Villapol S. Gastrointestinal symptoms associated with COVID19: impact on the gut microbiome. Translational Research. 2020; 226: 57-69.

[56] Marotz C, Belda-Ferre P, Ali F, Das P, Huang S, Cantrell K, et al. SARS-CoV-2 detection status associates with bacterial community composition in patients and the hospital environment. Microbiome. 2021; 9: 132.

[57] Kalantar-Zadeh K, Ward SA, Kalantar-Zadeh K, El-Omar EM. Considering the Effects of Microbiome and Diet on SARS-CoV2 Infection: Nanotechnology Roles. ACS Nano. 2020; 14: 51795182.

[58] Ciabattini A, Olivieri R, Lazzeri E, Medaglini D. Role of the Microbiota in the Modulation of Vaccine Immune Responses. Front Microbiol. 2019; 10: 1305.

[59] Soiza RL, Donaldson AIC, Myint PK. The pale evidence for treatment of iron-deficiency anaemia in older people. Therapeutic Advances in Drug Safety. 2018; 9: 259-261.

[60] McCall C, Wu H, Miyani B, Xagoraraki I. Identification of multiple potential viral diseases in a large urban center using wastewater surveillance. Water Research. 2020; 184: 116160.

[61] Lodder W, de Roda Husman AM. SARS-CoV-2 in wastewater: potential health risk, but also data source. Lancet Gastroenterol Hepatol. 2020; 5: 533-534.

Keywords: COVID-19; SARS-CoV-2; Immunity; Microbiota; Review

Send correspondence to: Amedeo Amedei, Department of Experimental and Clinical Medicine, University of Florence, 50134 Florence, Italy, SOD of Interdisciplinary Internal Medicine, Azienda Ospedaliera Universitaria Careggi (AOUC), 50134 Florence, Italy, E-mail: amedeo.amedei@unifi.it 